Journal of Computer Science 7 (4): 561-567, 2011

ISSN 1549-3636

(C) 2011 Science Publications

\title{
Topology Control Using Efficient Power Management
}

\author{
${ }^{1}$ S. Karunakaran and ${ }^{2} \mathrm{P}$. Thangaraj \\ ${ }^{1}$ Department of Computer Science and Engineering, Kongu Engineering College \\ Perundurai-638052, India \\ ${ }^{2}$ Department of Computer Science and Engineering, Bannari Amman Institute of technology \\ Sathyamanagalam, Erode, Tamilnadu, India
}

\begin{abstract}
Problem statement: Effective Topology control will improve the performance and the capacity of Mobile Ad-hoc networks by building reliable network structure. Approach: Power level is used as the basis for topology control since it reduces spatial interference while increasing the effective channel capacity and network lifetime. Results: In this study, we design a topology control using efficient power management algorithm using clustering. In this study, initially the cluster-head is selected based on the factors power level, stability and connectivity. Then, the connectivity of each node of the cluster with the cluster- head is checked. If the connectivity is less than a minimum connectivity threshold, then it is increased by increasing the power level. If the connectivity is higher than a maximum threshold, then the cluster-head reduces its power level. Conclusion/Recommendations: By simulation results, we show that the proposed topology control algorithm outperforms existing topology control algorithms in terms of by increased node life time and reduced power consumption.
\end{abstract}

Key words: Mobile Ad-Hoc Network (MANET), efficient power management, effective topology, power level, node life time, connectivity threshold, topology control algorithms, routing protocol, weighted clustering algorithm, power estimation

\section{INTRODUCTION}

Topology Control in MANET: A mobile ad-hoc network (MANET) comprises of a group of mobile host nodes which work without the help of centralized management or any of the pre-existing infrastructure to form a provisional network. A Mobile ad-hoc network (MANET) is formed when several wireless mobile nodes which posses restricted capability for communication work together (Sahin et al., 2010).

In mobile ad-hoc networks (MANETs), topology control is a key problem, due to the constant change in the underlying topology of the communication network. Ad-hoc networks are characterized by multi-hop wireless connectivity and the efficient topology control plays an important role (Zhao and Lloyd, 2006). The main intention of using the topology control in MANETs is to develop the capacity and performance in order to achieve desirable objectives by building specific topologies. A general method of topology control is to reduce the spatial interference by regulating the transmission power of every node which in turn increases the effective channel capacity and also the network lifetime.
In the ad-hoc networks, the topology control largely aims in allotting power levels to the nodes in order to maintain a specific topology like the requirement which needs the network to be connected. Further, the allotment of the transmission power to every node in the network aims in optimizing some of the functions of those powers. This is done mainly to reduce either the maximum power used by any of the node in the network (MinMax) or the average power used by the nodes (MinTotal) in the network (Jayakumar and Gopinath, 2008).

Topology control using clustering: One of the most fundamental ways to design a scalable ad-hoc network is by using the clustering technique. Network clustering is an important technique widely used in efficient MANETs network management, hierarchical routing protocol design, network modeling, Quality of Service, (Francis and Rajsingh, 2008) . In the clustering technique, the network is divided into subsets containing a group of nodes with the cluster-head placed at the midpoint of each cluster. A good clustering gives rise to a regular high level structure. It is easy to design an efficient energy conserving protocol for nodes in a clustered organization level when compared with the level of individual nodes.

Corresponding Author: S. Karunakaran, Department of Computer Science and Engineering, Kongu Engineering College, Perundurai-638052, Erode, Tamilnadu, India 
The use of subsets of the network nodes serving as backbone in supporting some functions is an efficient approach to manage the ad-hoc network topology. It elects a set of cluster-heads with every node in the network associated to the cluster- head. In the ad-hoc networks, the election of the cluster-head in the cluster reduces the topology maintenance factor.

In the wireless ad-hoc networks, the reduction in the power consumption in order to increase the network lifetime is the main goal of the topology control (Gupta and Pandey, 2008). Prolonging the lifetime of the network through energy aware node organization is highly desirable since it is battery powered (Xuan et al., 2007).

The inefficient use of the power and bandwidth by every node in communicating directly to another node can be solved by the process of partitioning the network into clusters. In each cluster the cluster-head will be the upper layer node and is responsible for the management of the cluster and coordination with the other clusters. There are several heuristic being proposed for the selection of the cluster-heads. In the proposed approach the cluster-head selection is based on our previous work (Karunakarn and Thangaraj, 2008), where the selection of the cluster-head is performed by the assignment of the weight value based on the factors: Power Level, Connectivity and Stability.

Related work: Adabi et al. (2008) proposed a new Distributed Score Based Clustering Algorithm (DSBCA) that considers the Battery Remaining, Number of Neighbors, Number of Members and Stability in order to calculate the node's score with a linear algorithm. After each node calculates its score independently, the neighbors of the node are notified. Each node selects one of its neighbors with the highest score to be its cluster-head and therefore the selection of cluster-heads is performed in a distributed manner with most recent information about current status of neighbor nodes. When compared with Weighted Clustering Algorithm and Distributed Weighted Clustering Algorithm in terms of number of clusters, number of re-affiliations and lifespan of nodes in the system, end-to-end throughput and overhead this algorithm has achieved the goals.

Alhunaity, (Zabian, 2007) analyzed different parameters of quality for the network models of base stations (BS) when the service messages have no delay. Four topologies star, ring, radial ring and fully connected are compared using identical quality of user service and conditional cost. Results have been obtained for different probabilities of failure in service message on one branch and fixed number of web channels. The results show that radial ring topology outperform better than the others with respect to both cost and hierarchy.

Zabian, (Alhunaity, 2006) presented a topology control mechanism and a routing protocol for Ad-Hoc wireless networks that chooses the shortest path given the lower power consumption. The power consumption in forwarding a packet depends on two factors number of hops and the packet size. The analytical results and simulation results gave the same results for power consumption in sending a packet taking in consideration only the power consumed in communication.

Chou and Suen (2006) proposed a Topologycontrol-based QoS Routing (TLQR) protocol, which is a distributed scheme to intelligently establish routes with guaranteed end-to-end bandwidth. They provide a simple residual-hop-count procedure to determine whether a node is capable to accept the new connection request or not. In addition, to enhance the network utilization an efficient power adjustment into the route discovery procedure is also incorporated.

Meraihi et al. (2004) proposed to deploy a controlled backbone in the ad- hoc environment using movable mobile routers. It concentrates on two fundamental problems: on the one hand, global connectivity of the network is investigated and on the other hand, to elaborate mechanisms that allow QoS support by setting an upper bound on the number of wireless hops. They describes the Mixed Integer Linear Programming (MILP) models for these deployment policies with respect to the constraints within these environments. The remaining work is to consider the parameters like signal power level and power management and dynamic mobile routers deployment.

Han et al. (2006) proposed a localized topology control algorithms for H-MANETs termed Topology Control with Power Estimation (TCPE). By using the technique of power estimation, TCPE directly collects the minimum transmission power among nodes for topology control without requiring any location, angle of- arrival, or global topology information. TCPE preserves the network connectivity and greatly improves the spatial reuse and power efficiency. TCPE also includes a topology maintenance mechanism to address mobility.

Fathi and Taheri (2010) proposed a new topology control protocols that extend the lifetime of dense adhoc networks while preserving connectivity, the ability for nodes to reach each other. These protocols conserve energy by identifying redundant nodes and turning their radios off. Cluster-based Energy Conservation (CEC) 
directly observes radio connectivity to determine redundancy nodes and so can be more aggressive at identifying duplication and more robust to radio fading. This protocol ensures that the connectivity in the network is maintained while consuming minimum energy.

Pradhan and Saadawi (2009) proposed a distributed power management algorithm that decides on an optimum coverage area for individual node to preserve network connectivity, reduce interference thus improve network performance with changing environment and network topology. In order to be strongly connected in the network, a node may increase its power indiscriminately causing interference. Since interference is one of the major problems in wireless network, the proposed algorithm will co-operatively reduce inter-node interference in the network. Uni-directional links are also a major source of interference as most of the routing protocol only utilizes bi-directional links. This algorithm attempt to prevent such links or if required convert them into bi-directional links.

Two topology control protocols that extend the lifetime of dense Ad-hoc networks while preserving connectivity and conserve energy by identifying redundant nodes and turning their radios off. Geographic Adaptive Fidelity (GAF) identifies redundant nodes by their physical location and a conservative estimate of radio range. Cluster-based Energy Conservation (CEC) directly observes radio connectivity to determine redundancy and so can be more aggressive at identifying duplication and more robust to radio fading. Experimental results show that their protocols are robust to variance in node mobility, radio propagation and node deployment density.

$\mathrm{Wu}$ and Dai (2006) reviewed the inconsistent information, which makes a node select too few logical neighbors and outdated information, which makes a node use too small a transmission range. They adapt a mobility-sensitive topology control method that extends many existing mobility insensitive protocols. Two mechanisms are introduced, consistent local views that avoid inconsistent information and delay and mobility management that tolerate outdated information.

Mousavi et al. (2007) explored that the major problem with Mobility aware distributed topology control protocol is the future distance predictor, which uses mobility prediction to estimate the future distance of neighboring nodes. The efficiency of this estimator varies in presence of different mobility models, sampling rates and different speed ranges. They introduce an adaptive mobility prediction method that uses learning automaton to estimate the coefficients of a simple adaptive filter in order to predict the future distance of two neighboring nodes.
The major problem with the existing protocols is the future distance predictor which uses mobility prediction to estimate the future distance of neighboring nodes. The efficiency of this estimator varies in presence of different mobility models, sampling rates and different speed ranges. This study introduces an adaptive mobility prediction method that uses learning automaton to estimate the coefficients of a simple adaptive filter in order to predict the future distance of two neighboring nodes.

\section{MATERIALS AND METHODS}

Consider a network of $\mathrm{m}$ cluster-heads, each having $n$ nodes in an area $\mathrm{A}$. If $\operatorname{Pi}(\mathrm{t})$ and $\sigma_{\mathrm{i}}(\mathrm{t})$ represent the transmitting power and connectivity of the clusterhead $\mathrm{i}$ in the network at time $\mathrm{t}$, then select $\mathrm{P}_{\mathrm{i}}(\mathrm{t})$ for cluster-head i $\forall 1,2,3, . ., \mathrm{n}$ subject to the following constraints.

The cluster-head should have at least minimum connectivity, $\sigma_{\text {imin }}(t)$, i.e. minimum acceptable number of neighbors with which the cluster-head has a bidirectional link with at any time t. The cluster-head and the cluster members should have bidirectional links:

$\sigma_{i}(t) \geq \sigma_{\text {imin }}(t)$ for node $i \forall 1,2, \ldots, n$.

For a packet from cluster-head $\mathrm{j}$ to cluster-head $\mathrm{i}$ to be correctly detected, Signal to Interference and Noise Ratio (SINR) must be greater than a threshold, $\gamma_{\mathrm{th}}$ :

$\mathrm{N} \rightarrow$ Set of transmitting nodes causing interference

$\mathrm{P}_{\mathrm{ki}} \rightarrow$ Received power level from node $\mathrm{k}$ to node $\mathrm{i}$

$\mathrm{P}_{0} \rightarrow$ Background noise

$$
\begin{aligned}
\operatorname{SINR}_{\mathrm{ji}}(\mathrm{t})= & \frac{\mathrm{P}_{\mathrm{ji}}(\mathrm{t})}{\mathrm{P}_{\mathrm{o}}+\Sigma \mathrm{P}_{\mathrm{ki}}(\mathrm{t})} \geq \gamma_{\mathrm{th}} \text { for cluster head } \mathrm{i} \forall 1,2, \ldots \mathrm{n}(3.2) \\
& \mathrm{k} \in \mathrm{N}, \mathrm{k} \notin \mathrm{j}
\end{aligned}
$$

The cluster-head should not transmit at a high level that it causes interference to other cluster-head in the neighborhood. The inter-cluster interference is minimized as shown in Eq. 3.3:

$$
\begin{gathered}
\operatorname{Min}\left[\mathrm{P}_{\circ}+\sum \mathrm{P}_{\mathrm{ki}}(\mathrm{t})\right] \text { for cluster head } i \forall 1,2 \ldots \mathrm{n} \\
\qquad \mathrm{k} \in \mathrm{N}, \mathrm{k} \notin \mathrm{j}
\end{gathered}
$$

If in a cluster, if the cluster-head has high connectivity with its nodes and other cluster-heads, then it can probably afford to decrease its power level and still maintain acceptable connectivity. Let $\sigma_{\text {imax }}(\mathrm{t})$ is the 
maximum number of neighbors allowed i.e. the upper acceptable connectivity threshold. This has an advantage of decreasing inter-node interference in the network:

$\sigma_{i}(t) \leq \sigma_{\text {imax }}(t)$ for cluster-head $i \forall 1,2 \ldots . . n$

The transmit power for the cluster-heads should be more than the minimum power level, $\mathrm{Pi}_{\min }$, but less than the maximum power level, $\mathrm{Pi}_{\max }$ defined by network and cluster-head power specifications:

$\mathrm{Pi}_{\text {min }} \leq \mathrm{P}_{\mathrm{i}}(\mathrm{t}) \leq \mathrm{Pi}_{\max }$ for cluster-head $\mathrm{i} \forall 1,2 \ldots, \mathrm{n}$

The proposed algorithm conserves cluster-head's battery capacity, $\mathrm{C}_{\mathrm{i}}(\mathrm{t})$, which is one of the important design considerations for mobile ad-hoc networks. The proposed algorithm allows the nodes to increase their power level, only if their battery power is higher than the critical battery power level $\mathrm{C}_{\text {icritical }}$ :

$\mathrm{C}_{\mathrm{i}}(\mathrm{t}) \geq \mathrm{C}_{\text {icritical }}$ for node $\mathrm{i} \forall 1,2 \ldots . \mathrm{n}$

Increasing the power level: Let the power level and maximum power level are denoted by $\mathrm{PL}_{\mathrm{i}}$ and $\mathrm{PL}_{\mathrm{imax}}$. The node connectivity and the minimum node connectivity is represented as $\sigma_{i}$ and $\sigma_{\text {imin }}$.The clusterhead increases the power level and establish a bidirectional link using the procedure shown below:

If $\sigma_{\mathrm{i}}<\sigma_{\text {imin }}$ and $\mathrm{PL}_{\mathrm{i}}<\mathrm{PL}_{\mathrm{imax}}$, then

the node will improve its connectivity by increasing its power level.

End if

If there are uni-directional links from the neighbor nodes, then

$\mathrm{PL}_{\mathrm{i}}=\mathrm{PL}_{\mathrm{i}}+\alpha$

cluster-head constructs bidirectional link with those

nodes

Else

$\mathrm{PL}_{\mathrm{i}}=\mathrm{PL}_{\mathrm{i}}+\alpha$

cluster-head constructs bidirectional link with 2 hop non

neighbor nodes

End if

Node sends a High Power Request HPreq to all 2 hop neighbors

If node $i$ receives a HPreq and if $\sigma_{i}>\sigma_{\text {imin }}$ then

4.1 $\mathrm{PL}_{\mathrm{i}}=\mathrm{PL}_{\mathrm{i}}+\alpha$

End if
Decreasing the power level: The cluster-head decreases the power level using the procedure shown below:

If the cluster- head moves into a dense area i.e., $\sigma_{i}$ $>\sigma_{\text {imax }}$ then

$\mathrm{PL}_{\mathrm{i}}=\mathrm{PL}_{\mathrm{i}}-\alpha$

End if

If the battery capacity $\mathrm{C}_{\mathrm{i}}<\mathrm{C}_{\text {icritical }}$, then

$\mathrm{PL}_{\mathrm{i}}=\mathrm{PL}_{\mathrm{i}}-\alpha$

End if

If node is suffering from interference, then

Node will send LPreq to other nodes after setting the

TTL, req TTL

End if

If a node receives a LPreq and if $\sigma_{i}>\sigma_{\text {imin }}$, then

$\mathrm{PL}_{\mathrm{i}}=\mathrm{PL}_{\mathrm{i}}-\alpha$

$\sigma_{i}$ is checked frequently after a time delay of $\tau_{\text {short_delay }}$

or long time delay, $\tau_{\text {long_delay }}$

End if

If $\mathrm{C}_{\mathrm{i}}<\mathrm{C}_{\text {icritical }}$, then

$\mathrm{PL}_{\mathrm{i}}=\mathrm{PL}_{\mathrm{i}}-\alpha$

End if.

\section{RESULTS AND DISCUSSION}

Simulation model and parameters: The following (Table 1) summarizes the simulation parameters used.

Performance metrics: The performance of the proposed TC-EPM algorithm is evaluated using Network simulator (Ns-2). Number of alive nodes and power consumption are used as metrics to compare the performance of TC-EPM with CEC, CLTC (Chung et al., 2004), ECEC (Fathi and Taheri, 2010).

Number of traffic sources alive: Figure 1 shows the number of nodes alive with respect to the time in seconds. The simulation time varied from 100 to 500 seconds and the corresponding number of nodes alive is absorbed. All the four protocols under comparison able to keep most nodes alive upto $200 \mathrm{sec}$. After that the alive number of nodes varies depending on the effectiveness of the protocol in its energy conservation and maintaining node connectivity. ECEC only about $75 \%$ of the nodes are alive at the time 500 . This is because to preserve energy it power off the nodes other than cluster-head and gateway. CEC protocol conserves energy by identifying redundant nodes and turning their radios off while maintaining minimum connectivity among the other nodes. 


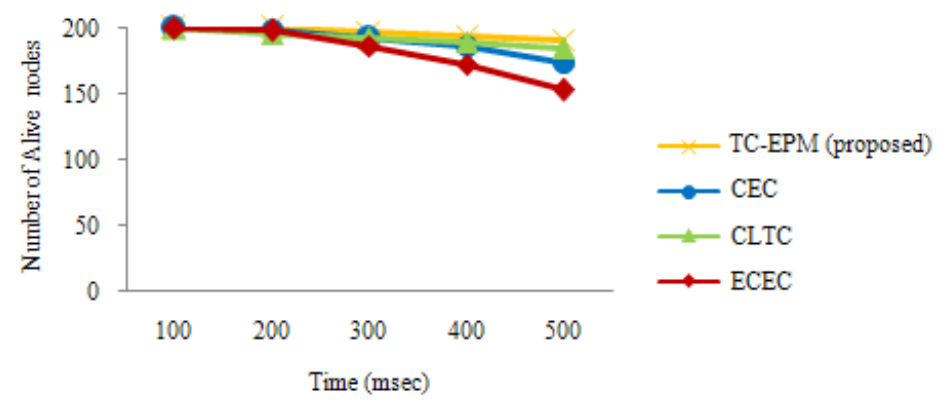

Fig. 1: Number of alive nodes with respect to time

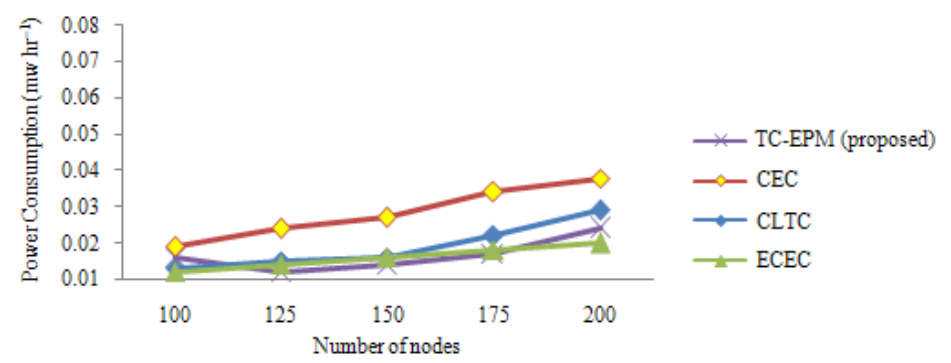

Fig. 2: Power consumption with respect to number of nodes

\begin{tabular}{ll} 
Table 1: & \\
\hline No. of nodes & 200 \\
\hline Area Size & $1500 \times 1000$ \\
Mac & $802.11 \mathrm{~b}$ \\
Traffic Source & $\mathrm{CBR}$ \\
Simulation time & $100-500 \mathrm{~ms}$ \\
Packet Size & 512 \\
Transmit Power & $0.360 \mathrm{w}$ \\
Receiving Power & $0.395 \mathrm{w}$ \\
Idle Power & $0.335 \mathrm{w}$ \\
Initial Energy & $0.5 \mathrm{~J}$ \\
Routing Protocol & AWCBRP \\
Speed & $5 \mathrm{~m} \mathrm{~s}^{-1}$ \\
\hline
\end{tabular}

CLTC balances energy use more evenly among nodes than CEC. At least $92 \%$ of CLTC nodes are alive except the scenario with at time of 100 where all the nodes alive. CLTC is more effective at balancing energy because of its connectivity measurement based approach. High mobility in CEC causes more frequent cluster formations and more overhead. With CLTC, high mobility helps balance energy use because changes in node location cause active node re-election within grids. About $25 \%$ of the traffic sources in ECEC are dead at time 500 , about $87 \%$ and $82 \%$ of the traffic sources are still alive in CEC and CLTC, respectively and more than $95 \%$ of the traffic sources are still alive in TC-EPM. The basic CEC algorithm does not perform as well as the CLTC, but it still performs $14 \%$ better than ECEC. The frequent change of cluster-heads will result in poor connectivity. Hence TC-EPM elects a consistent cluster-head to check and regulate the minimum connectivity power level to its cluster members. By this efficient power adjustment, improved topology is attained in TC-EPM that outperforms CLTC, CEC and ECEC to keeps more number of nodes alive in the network.

Power consumption: Figure 2 shows the power consumption in terms of $\mathrm{mw} / \mathrm{hr}$ with respect to the number of nodes. The number of nodes varied from 100-200 seconds and the corresponding power consumption is absorbed. The power consumption is less than 0.02 for all the protocols under consideration of 100 numbers of nodes. For 175 nodes the power consumption of CLTC and CEC increased by 0.005 and 0.017 respectively when compared to TC-EPM and ECEC. CEC shows high power consumption for all the nodes from 100-200 since it divides the network into overlapping clusters and utilizes more energy to keep its gateway nodes alive. Centralized approach able to achieve strong connectivity but suffers from scalability problems. In contrast, a distributed approach like CEC, although scalable, lacks strong connectivity guarantees and consumes more power. CLTC is a hybrid topology control framework that achieves both scalability and strong connectivity and show improved power consumption than CEC. Both CLTC and ECEC shows 
almost the same performance up to 150 nodes. But for nodes 175 and 200 ECEC utilizes .004 and .009 less power than CLTC. The proposed TC-EPM initially for 100 nodes consumes $25 \%$ more power than CLTC since initially more packets exchanged to select effective cluster- head with longer life time. For nodes 125-175 TC-EPM illustrates improved performance than ECEC with around $10 \%$ less power consumption. When the number of nodes increased as 200 ECEC consumes $20 \%$ less power than TC-EPM and outperforms CEC, CLTC and TC-EPM.

\section{CONCLUSION}

An efficient topology control algorithm is proposed that uses power level as the basis. In this technique, topology control is maintained within each cluster. Initially, the cluster-head is selected based on factors power level, stability and connectivity. After the cluster-head selection, the connectivity of each node of the cluster with the cluster-head is checked. If the connectivity is low then the connectivity is increased by increasing the power level. In a cluster, cluster-head which is incharge of a dense area will experience low inter node interference. If there are any unidirectional links in the network then the cluster-head will form bidirectional link with it. If there are no unidirectional links, then the cluster-head will start linking up with the nodes that are not its direct neighbors. If the connectivity is higher than a threshold, then the cluster-head reduces the power level. Thus, by proper adjustment of the transmission power, efficient topology control can be attained. The simulation results shown that the proposed topology control algorithm achieves improved node life time with reduced power consumption.

\section{REFERENCES}

Adabi, S., S. Jabbehdari, R. Amirmasoud and S. Adabi, 2008. A novel distributed clustering algorithm for mobile ad-hoc networks. J. Comput. Sci., 4: 161-166. DOI: $10.3844 /$ jcssp.2008.161.166

Alhunaity, M.F., 2006. Comparative study between various topological models of base stations in cellular mobile radio communication. Am. J. Applied Sci., 3: 1711-1714. DOI: 10.3844/ajassp.2006.1711.1714

Chou, C.F. and H.P. Suen, 2006. Topology-Controlbased QoS Routing (TLQR) in wireless Ad Hoc networks. Proceedings of the 17th International Symposium on Personal, Indoor and Mobile Radio Communications, Sept. 11-14, IEEE Xplore, Helsinki, pp: 1-5. DOI: 10.1109/PIMRC.2006.254168
Fathi, A. and H. Taheri, 2010. Enhance topology Control Protocol(ECEC) to conserve energy based clustering in wireless ad hoc networks. Proceedings of the 3rd IEEE International Conference on Computer Science and Information Technology, July 9-11, IEEE Xplore, Chengdu, pp: 356-360. DOI: 10.1109/ICCSIT.2010.5564463

Francis, S.J. and E.B. Rajsingh, 2008. Performance analysis of clustering protocols in mobile ad hoc networks. J. Comput. Sci., 4: 192-204. DOI: 10.3844/jcssp.2008.192.204

Gupta, V. and R. Pandey, 2008. Data fusion and topology control in wireless sensor networks. WSEAS Trans. Signal Process., 4: 150-172.

Han, X., C.C. Shen and Z. Huang, 2006. Adaptive Topology Control for Heterogeneous Mobile Ad hoc Networks Using Power Estimation. Proceedings of the IEEE Wireless Communications and Networking Conference, Apr. 3-6, IEEE Xplore, Las Vages, NV., pp: 392-399. DOI: 10.1109/WCNC.2006.1683497

Jayakumar, G. and G. Gopinath, 2008. Performance comparison of two on-demand routing protocols for ad-hoc networks based on random way point mobility model. Am. J. Applied Sci., 5: 659-664. DOI: 10.3844/ajassp.2008.659.664

Karunakarn, S. and P. Thangaraj, 2008. An Adaptive Weighted Cluster Based Routing (AWCBRP) Protocol for Mobile Ad-hoc Networks. WSEAS Trans. Commun., 7: 248-257.

Meraihi, R., G.L. Grand, N. Puech, M. Riguidel and S. Tohme, 2004. Improving ad hoc network performance with backbone topology control. Proceedings of the IEEE 60th Vehicular Technology Conference, Sept. 26-29, IEEE Xplore, France, $\quad$ pp: 2829-2833. DOI: 10.1109/VETECF.2004.1400575

Mousavi, S.M., H.R. Rabiee and M. Moshref, 2007. Mobility aware distributed topology control in mobile ad-hoc networks with model based adaptive mobility prediction. Proceedings of the 3rd IEEE International Conference on Wireless and Mobile Computing, Networking and Communications, Oct. 8-10, IEEE Xplore, White Plains, NY., pp: 86-86. DOI: 10.1109/WIMOB.2007.4390880

Pradhan, N. and T. Saadawi, 2009. Topology control using distributed power management algorithm for mobile ad hoc networks. Proceedings of the 17th International Conference on Software, Telecommunications and Computer Networks, Sept. 24-26, IEEE Xplore, Hvar, pp: 317-322. 
Sahin, C.S., S. Gundry, E. Urrea, M.U. Uyar and M. Conner et al., 2010. Convergence analysis of genetic algorithms for topology control in MANETs. Proceedings of the IEEE Sarnoff Symposium, Apr. 12-14, IEEE Xplore, Princeton, NJ., $\quad$ pp: $1-5 . \quad$ DOI: 10.1109/SARNOF.2010.5469783

Wu, J. and F. Dai, 2006. Mobility-sensitive topology control in mobile ad hoc networks. IEEE Trans. Parall. Distrib. Syst., 17: 522-535. DOI: 10.1109/TPDS.2006.73

Xuan, Y., Wang, Forrest and S. Bao, 2007. An entropybased weighted clustering algorithm and its optimization for ad hoc networks. Proceedings of the 3rd IEEE International Conference on Wireless and Mobile Computing, Networking and Communications, Oct. 8-10, IEEE Xplore, White Plains, NY., pp: 56-56. DOI: 10.1109/WIMOB.2007.4390850
Zabian, A., 2007. Topology discovering and power saving mechanism for routing in a tree of ad-hoc wireless networks. J. Comput. Sci., 3: 646-657. DOI: $10.3844 /$ jessp.2007.646.657

Zhao, L. and E.L. Lloyd, 2006. Distributed topology control for stationary and mobile ad hoc networks. Proceedings of the IEEE International Conference on Mobile Adhoc and Sensor Systems, (MOBHOC'06) IEEE Xplore, Vancouver, BC., pp: 521-524. DOI: 10.1109/MOBHOC.2006.278600 